\title{
Emergence of an Onion-like Network in Surface Growth and Its Strong Robustness
}

\author{
Yukio Hayashi* and Yuki Tanaka \\ Japan Advanced Institute of Science and Technology, \\ Ishikawa, 923-1292, Japan
}

(Dated: May 14, 2019)

\begin{abstract}
We numerically investigate that optimal robust onion-like networks can emerge even with the constraint of surface growth in supposing a spatially embedded transportation or communication system. To be onion-like, moderately long links are necessary in the attachment through intermediations inspired from a social organization theory.
\end{abstract}

PACS numbers: 89.20.-a, 05.65.+b, 05.45.Df, 02.60.-x

Keywords: Complex Network Science; Optimal Robust Structure; Generation Algorithm; Surface Growth; Intermediation

*Electronic address: yhayashi@jaist.ac.jp 


\section{INTRODUCTION}

It was found that onion-like networks with positive degree-degree correlations have the optimal tolerance of connectivity against attacks [1,2] in the state-of-the-art Network Science (at a boundary area of physics and computer science). In the network, onion-like structure is visualized by links between similar degree nodes because of the positive correlations, when nodes are set on concentric circles arranged in decreasing order of degrees from core to peripheral. Unfortunately, real technological networks (Internet or World-Wide-Web) are

vulnerable with negative correlations [3]. While there exist rewiring methods [2, 4] for a network in order to enhance the correlations positively and to be onion-like, they are not realistic. Because many already connected relations are discarded.

Recently, incrementally growing methods have been proposed for constructing an onionlike network $[5,6]$. One is based on copying and adding shortcut links [5]. Another is based on intermediations [6] inspired from some case studies in an organization theory [7], in which the importance of long-distance relations has been suggested for both robustness of connectivity and efficiency of path in the networks such as supply chain and business collaboration. The established connections via intermediations probably work well for managing crossborder operations. The copying [5] is a modification of the duplication-divergence model [8] inspired from a biologically growing mechanism. Moreover, it has been shown that onionlike networks are obtained even with a constraint of surface growth in the method based on copying and adding shortcut links [9]. By the constraint, the position of new node is limited on surface, however such a spatial embedding is rather natural such as in transportation or communication systems. In this paper, we investigate whether or not an onion-like network emerges in the growing method based on intermediations, when it is spatially embedded with a constraint of surface growth.

\section{HOW TO CONSTRUCT A NETWORK}

\section{A. Incrementally growing method}

We briefly survey the incrementally growing methods based on intermediations (MED) $[6,10]$ for constructing onion-like networks. From an initial configuration, e.g. a complete graph $K_{m}$ of $m$ nodes, a new node is added and connect to existing nodes with even number 
$m$ links at each time step. In the methods, a pair of attachments is repeatedly performed for the number of links. In other words, the destination nodes of $m / 2$ links are decided by one rule: random attachment [11], and the remaining them of $m / 2$ links are decided by another rule: attachment by MED. Either of the following MED-kmin and MED-rand is applied, the number of links emanated from a new node is $m / 2+m / 2=m$ at each time step.

MED-kmin One of the pair is chosen uniformly at random (u.a.r) from the existing nodes by random attachment. As another of it, a node with the minimum degree is chosen by intermediations in $\mu$ hops form its randomly chosen pair node. Intermediations in $\mu$ hops mean attachments to the $(\mu+1)$-th neighbors. $\mu \geq 0$ is a small integer.

MED-rand One of the pair is the same as MED-kmin. For another of it, instead of selecting the node with the minimum degree in MED-kmin, a node is chosen u.a.r in the $(\mu+1)$-th neighbors from its randomly chosen pair node.

For prohibited multi-links, choosing other node is tried. Intuitively, random attachment contributes to enhance the correlations between large degree nodes, since older nodes tend to get more links by random attachment [11] and to connect each other. While another attachment by MED contributes to enhance the correlations between small degree nodes, since it often happens that the degree of the $(\mu+1)$-th neighbor is small and the degree $m$ of new node is the minimum in the network. However, these roles are exchanged in the case with a constraint of surface growth as mentioned later.

\section{B. Constraint of surface growth}

Some most important classes of surface growth include Diffusion-Limited Aggregation (DLA) [12], Invasion Percolation (IP) [13], and Eden growth [14], which can be used as a basis for understanding a wide range of pattern-formation phenomena with (e.g. fractal) disorderly growth. Thus, as similar to the copying network [9], we consider the typical diffusive growth on surface, whose position of new node is determined by DLA, IP, or Eden model on a square lattice.

DLA model It means a dendric extension in technological or social network system. 


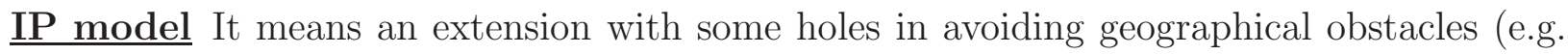
mountain or rivers) for the network construction.

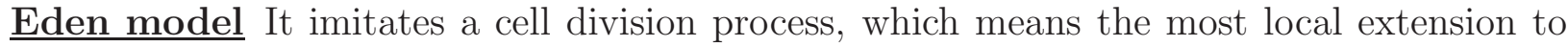
neighbor space in a simple way.

After determining the position of new node by DLA, IP, or Eden model, it connect to the existing nodes as shown in Fig. 1. Showing the rotated cases by $90^{\circ}, 180^{\circ}$, and $270^{\circ}$ are omitted because of the same. The shaded circles and arrows denote candidates of the position of new node and its links, while the dashed circle denotes a prohibited case because of lack of contact nodes less than $m / 2$. The open circles denote already existing nodes in the network. The contact of new node to surface corresponds to a random attachment, since the position of new node on surface is randomly chosen according to DLA, IP, or Eden model. As random attachment on surface, the destinations of $m / 2$ links are chosen from the neighbors (at least 2 and at most 7 ) indicated by arrowheads. We set $m=4$, because $m \geq 4$ is necessary to construct onion-like networks through intermediations without surface growth [6]. Thus, it is the constraint for linking or forming a topological structure that attached nodes of one side of pair are not freely chosen uniformly at random, but chosen from only surface. In addition, according to another attachment in MED-kmin or MED-rand, destinations of $m / 2$ links are chosen in the $(\mu+1)$-th neighbors from the pairs on surface. If there is no $(\mu+1)$-th neighbor, then the furthest node from its pair is chosen for a link. Note that links between a new node and the above neighbors make bridges whose lengths are more than unit length (one hop between contact nodes) of square lattice. Such constructed network is not a planar graph, however the limitation of planarity is hardly required in transportation or communication systems.

\section{EMERGENCE OF ONION-LIKE NETWORKS}

We use two measures of robustness index $R$ and assortativity $r$ for investigating the emergence of onion-like networks. The robustness index $1 / N \leq R \leq 0.5$ [2] is defined

as $R \stackrel{\text { def }}{=} \sum_{q=1 / N}^{1} S(q) / N$, where $S(q)$ denotes the number of nodes included in the giant component (as the largest connected cluster) after removing $q N$ nodes, $q$ is a fraction of removed nodes. In this paper, we consider malicious attacks with recalculation of the largest 


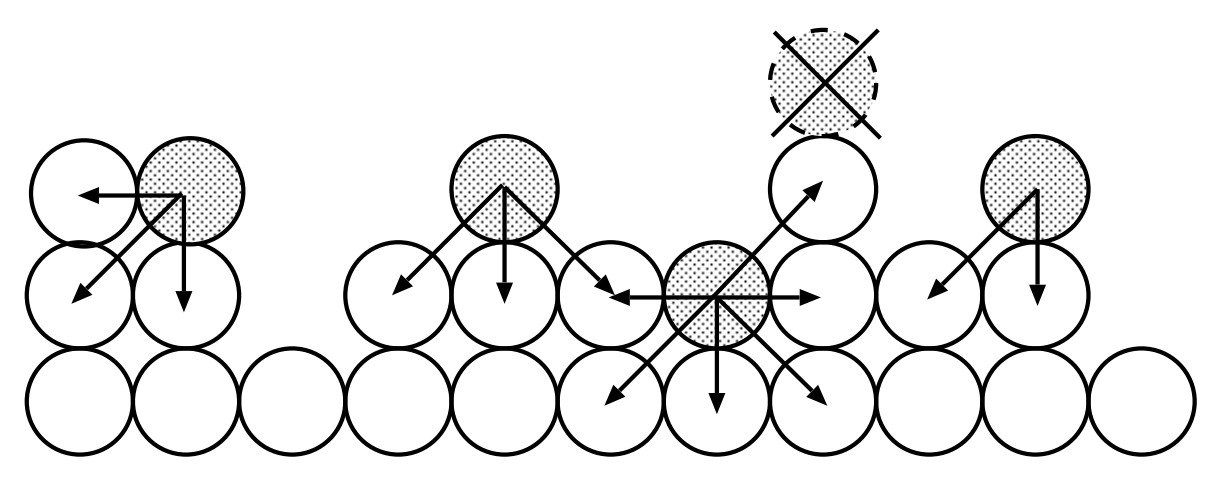

FIG. 1: Candidate positions of new node on surface.

degree node. For the measure of degree-degree correlations, as the Pearson correlation coefficient for degrees [3], the assortativity $-1 \leq r \leq 1$ is defined as

$$
r \stackrel{\text { def }}{=} \frac{4 M \sum_{e}\left(k_{e} k_{e}^{\prime}\right)-\left[\sum_{e}\left(k_{e}+k_{e}^{\prime}\right)\right]^{2}}{2 M \sum_{e}\left(k_{e}^{2}+k_{e}^{\prime 2}\right)-\left[\sum_{e}\left(k_{e}+k_{e}^{\prime}\right)\right]^{2}},
$$

where $k_{e}$ and $k_{e}^{\prime}$ denote degrees at end-nodes of link $e, M$ is the total number of links. When both $R$ and $r$ are large, the network is onion-like. Although there is no exact criterion to be onion-like, we investigate it in numerical comparison. The following results are averaged over 100 realizations of generated networks from the initial $K_{m}$ at the origin $(0,0)$ of square lattice.

Figure 2 shows the results for the growing method of MED-kmin. The robustness is almost constantly strong around $R>0.37$ except the cases of $\mu=0,1$ (purple and green lines). Similar robustness is obtained in the three models of surface growth. For the degreedegree correlations, only the cases of $\mu=6$ (red line) by DLA model and $\mu=5,6$ (blue and red lines) by IP model have $r>0.36$. The case of $\mu=6$ (red line) by Eden model have slightly small $R \approx 0.3$. In other cases, $r$ is small around $0.1 \sim 0.2$. Here, these $R \approx 0.37$ and $r \approx 0.36$ are the same level in the original method of MED-kmin without surface constraint $[6,10]$.

Figure 3 shows the results for the growing method of MED-rand. The robustness is also strong around $R>0.34$ except the cases of $\mu=0,1,2$ (purple, green, light blue lines). The robustness becomes larger as $\mu$ is larger in all three models of surface growth, the ordering of robustness by slight differences is Eden, IP, and DLA models from bottom to top. For the degree-degree correlations, the cases of $\mu=4,5,6$ (yellow, blue, and red lines) have $r>0.25$. The case of $\mu=0$ (purple line) are almost no correlation of $r \approx 0$. Here, these $R \approx 0.34$ and 
Robustness
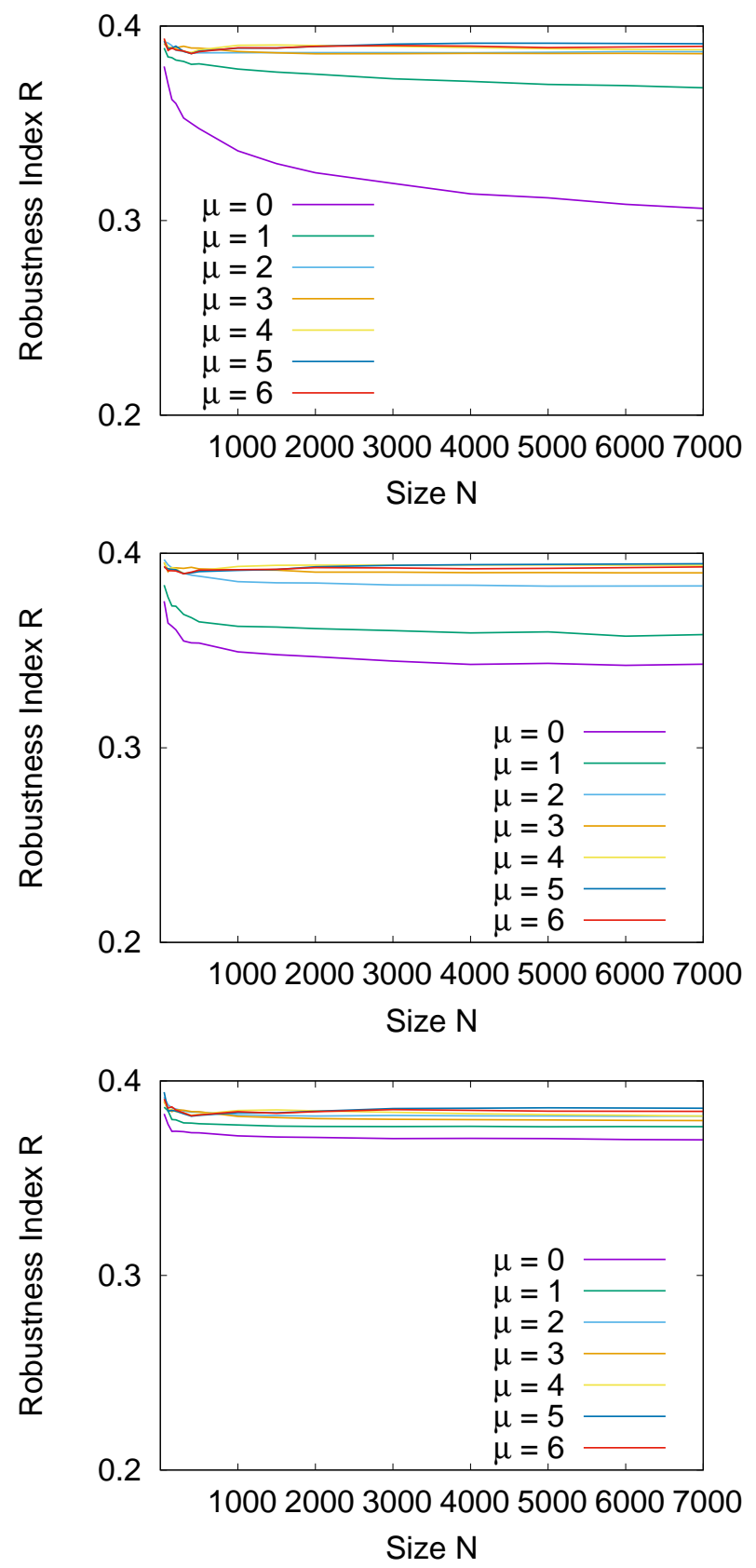

Correlation
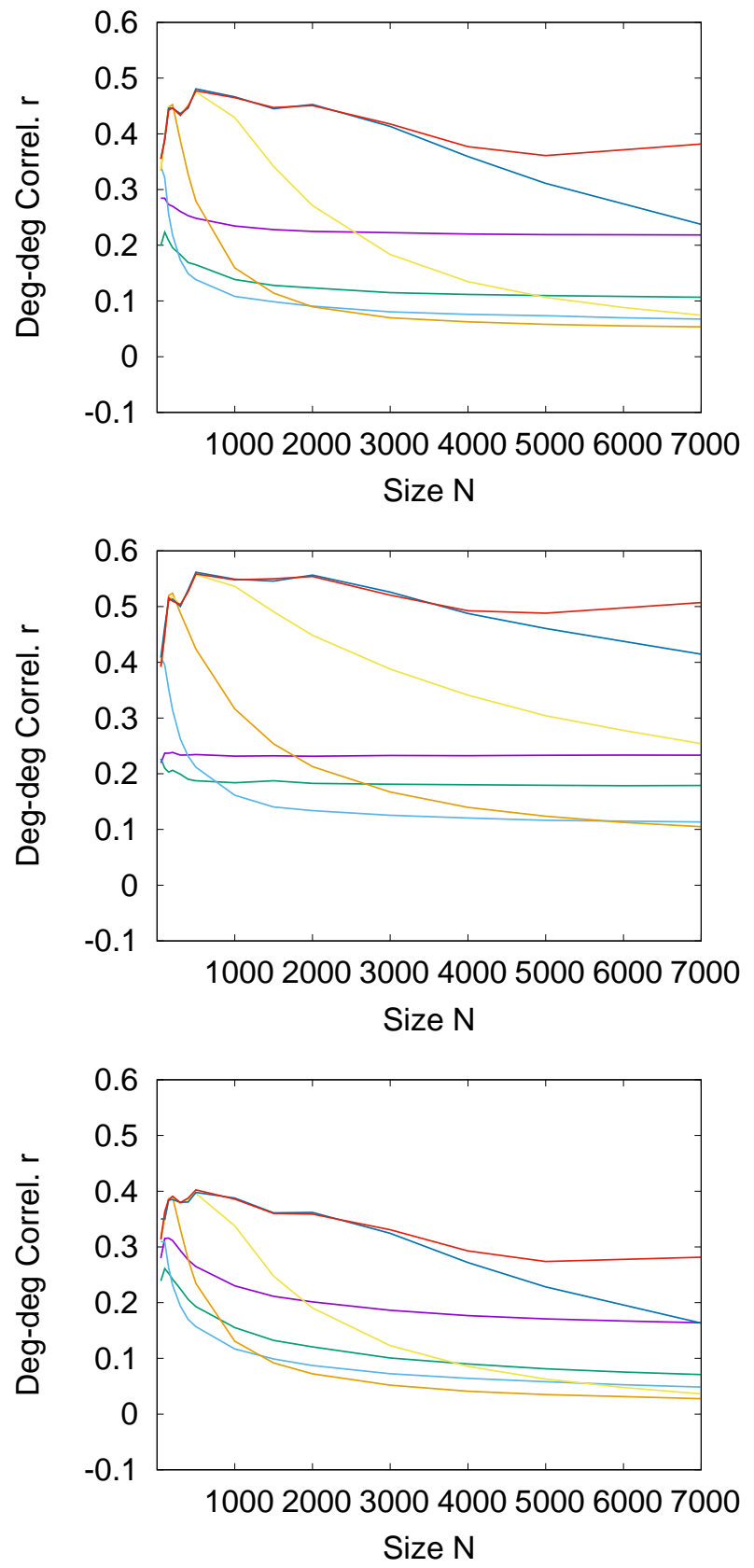

FIG. 2: Results for the method of MED-kmin with surface constraint by DLA(Top), IP(Middle), and Eden(Bottom) models.

$r \approx 0.25$ are the same level in the original method of MED-rand without surface constraint $[6,10]$. Thus, for the methods of MED-kmin and MED-rand of $\mu=6$ intermadiations, onion-like networks emerge with large $R$ and $r$ in the constraint of surface growth. Even if the network after growing until each size $N$ is rewired by Wu-Holme method [4], the 
robustness is similar around $R \approx 0.38 \sim 0.39$. Note that the copying networks with surface constraint have $R \approx 0.27$ and $r \approx 0.3$ [9], however the robustness is weaker as $R<0.2 \mathrm{in}$ early stage of $N<1000$.

Robustness
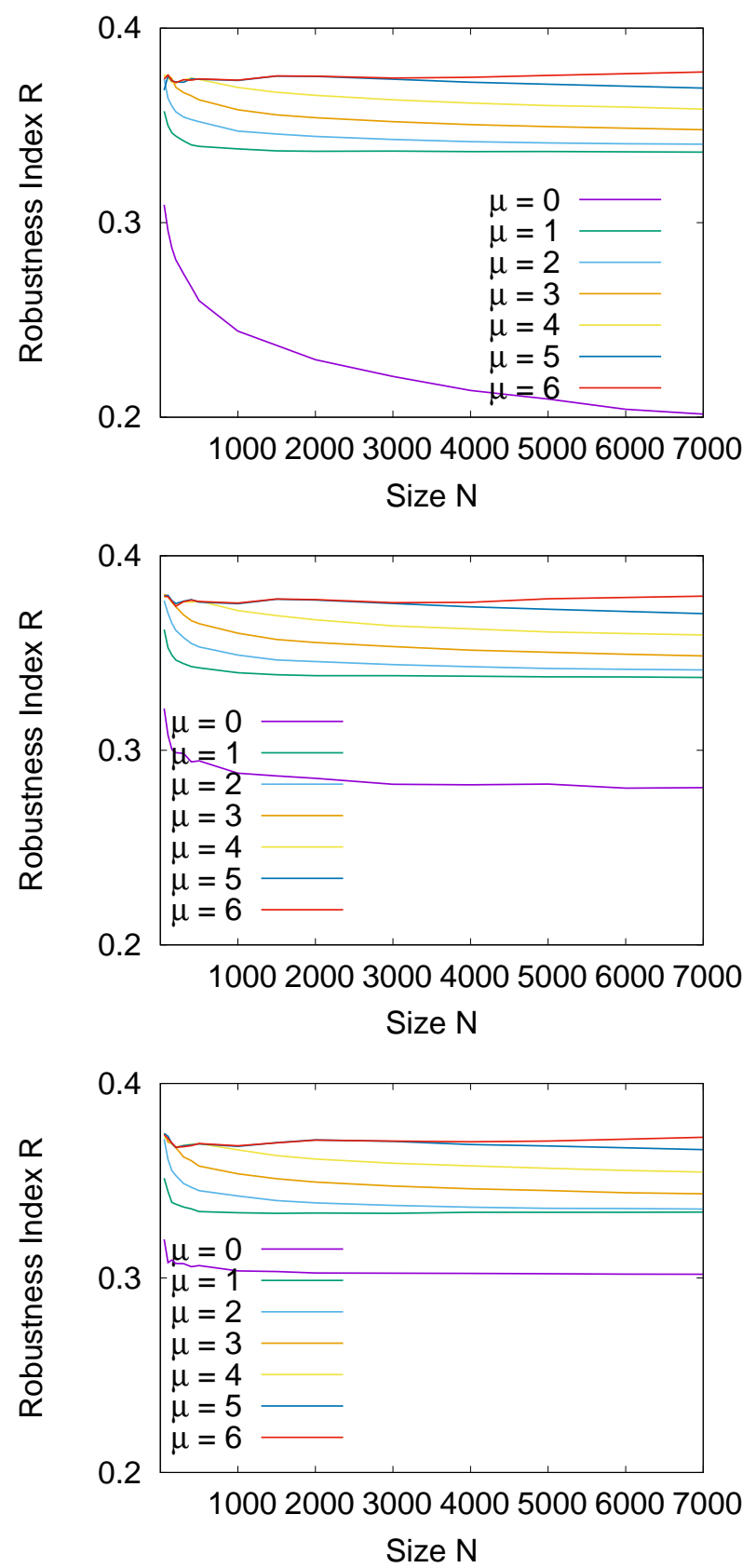

Correlation
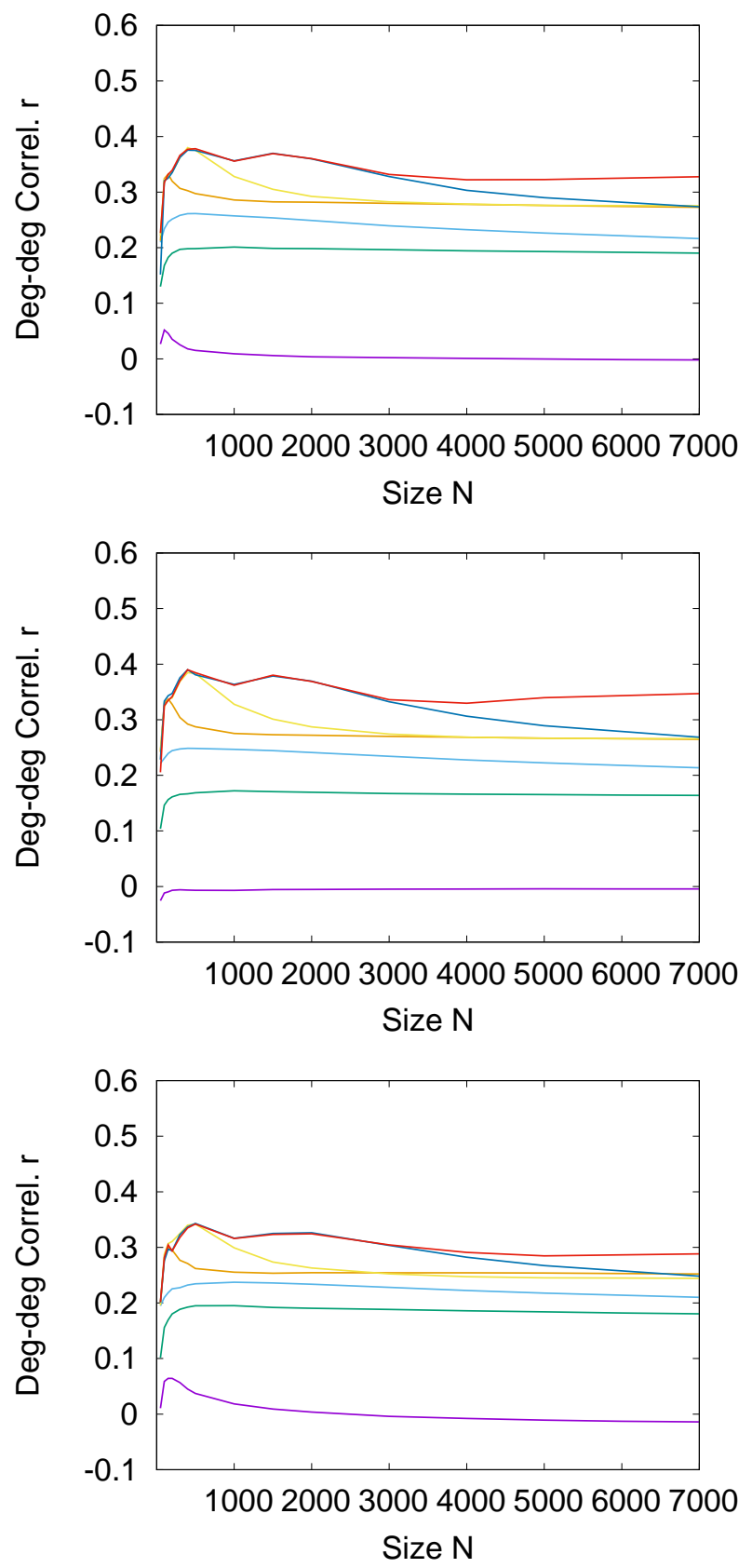

FIG. 3: Results for the method of MED-rand with surface constraint by DLA(Top), IP(Middle), and Eden(Bottom) models.

Moreover, we consider the reason why onion-like networks emerge in the methods. Figure 
4 shows the average degree of the nearest neighbors $(\mathrm{ADNN})\left\langle k_{n n}\right\rangle(k)$ as a function of $k$. As the slope $\Delta\left\langle k_{n n}\right\rangle / \Delta k$ close to one, similar degree nodes tend to connect, then the degree-degree correlations are enhanced. In contrast, as negative one, the correlations are inhibited. In the method of MED-kmin of $\mu=6$ (red line in the left of Fig. 4), positive slopes are dominant, while there are plateaus which weaken the correlations in other cases of $\mu=0,1, \ldots, 5$ (purple $\sim$ blue lines). The plateaus at $\left\langle k_{n n}\right\rangle(k) \approx 8$ which is corresponded to the maximum contacts 8 in random attachment are longer as $\mu$ is smaller. Similarly, in the method of MED-rand of $\mu=6$ (red line in the right of Fig. 4), positive slopes are dominant. Mixed positive and negative slopes exist in other cases of $\mu=0,1, \ldots, 5$. In particular, the slope is almost flat in the case of $\mu=0$ (purple line), and the flat part occupies about half of the line in the cases of $\mu=1,2$ (green and light blue lines). These length of plateau or frequency of negative slops correspond to the ordering of suppressed $r$ values in Figs. 2,3. We remark that the maximum degree in the method of MED-rand is larger than that in the method of MED-kmin.

The strong correlations shown by $\left\langle k_{n n}\right\rangle$ in Fig. 4 correspond to the existence of inner core of large degree nodes in the network with surface constraint by DLA(Top), IP(Middle), and Eden(Bottom) models. In Fig. 5, colors of nodes are assigned for each range of degrees; 4: white, 5, 6, 7: yellow, 8, 9, 10: green, 11,12, 13: gray, 14, ., 19: brown, and 20 over: red. Blue line denotes a link. In the method of MED-kmin, there exists no core of large degree nodes for intermediations of $\mu=0,1$ in Fig. 5(a)(b), however it emerges as a gray area for $\mu=5,6$ in Fig. 5(c)(d). It is difficult to see any difference from the visualization, although $\left\langle k_{n n}\right\rangle$ in Fig. 4 (blue and red lines) slightly differs for $\mu=5$ and $\mu=6$. Thus, moderately long links through intermediations are necessary in order to connect from a new node on surface to the core. We should remark that older nodes are closer to the core, and get links by intermediation attachment, while newer nodes are closer to surface, and get links by random attachment, mainly. The roles of enhancing the correlations for large nodes and small nodes are exchanged from them in the original method $[6,10]$ without constraint of surface growth.

In the method of MED-rand, large degree (brown and red) nodes appear in the inner core as shown in Fig. 5(a)-(d). There are several common features for MED-kmin and MED-rand. In the case by IP model, the position of core (gray or brown) with concentrated links is not determined in advance but by chance as shown at the middle in Fig. 5(a)-(d). 
MED-kmin
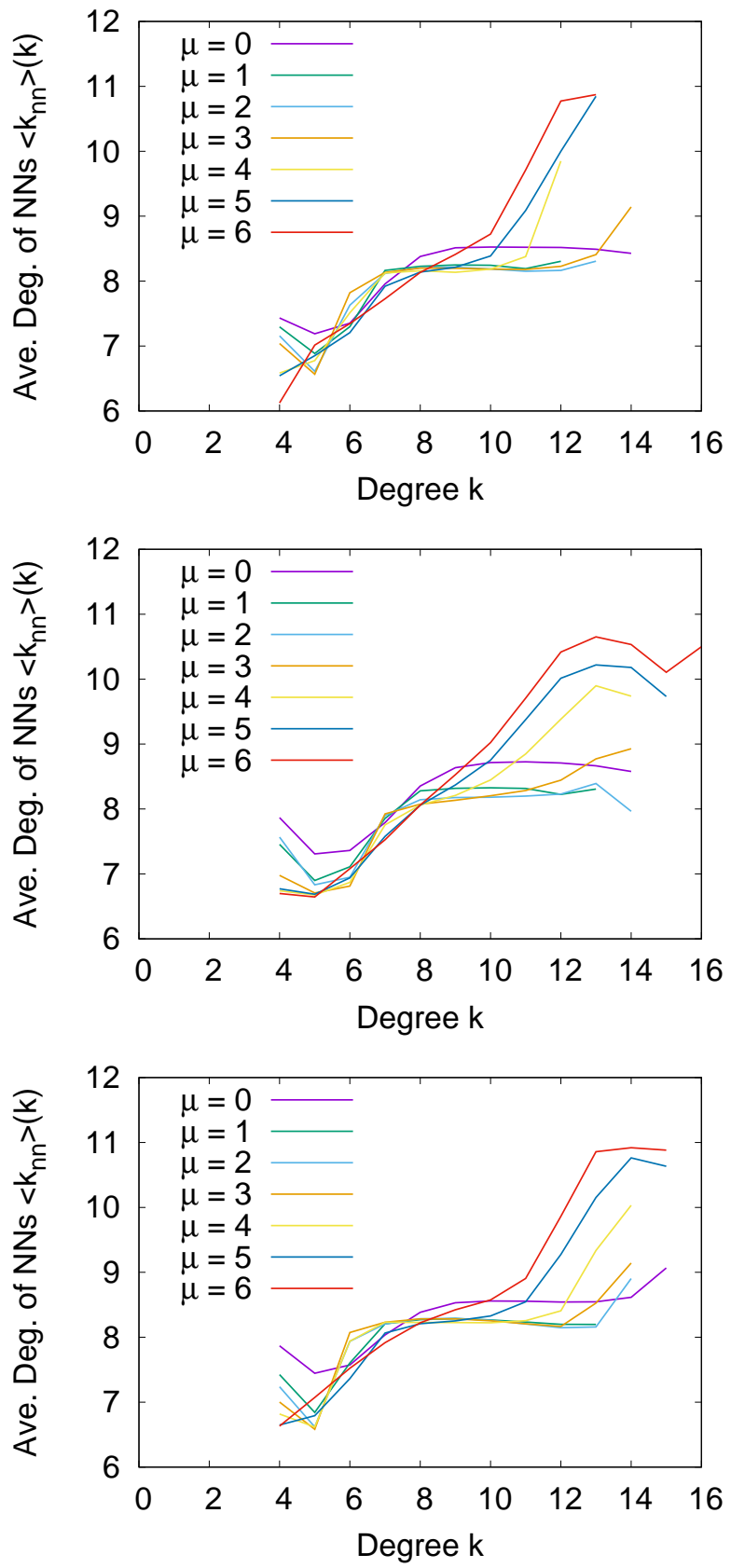

MED-rand
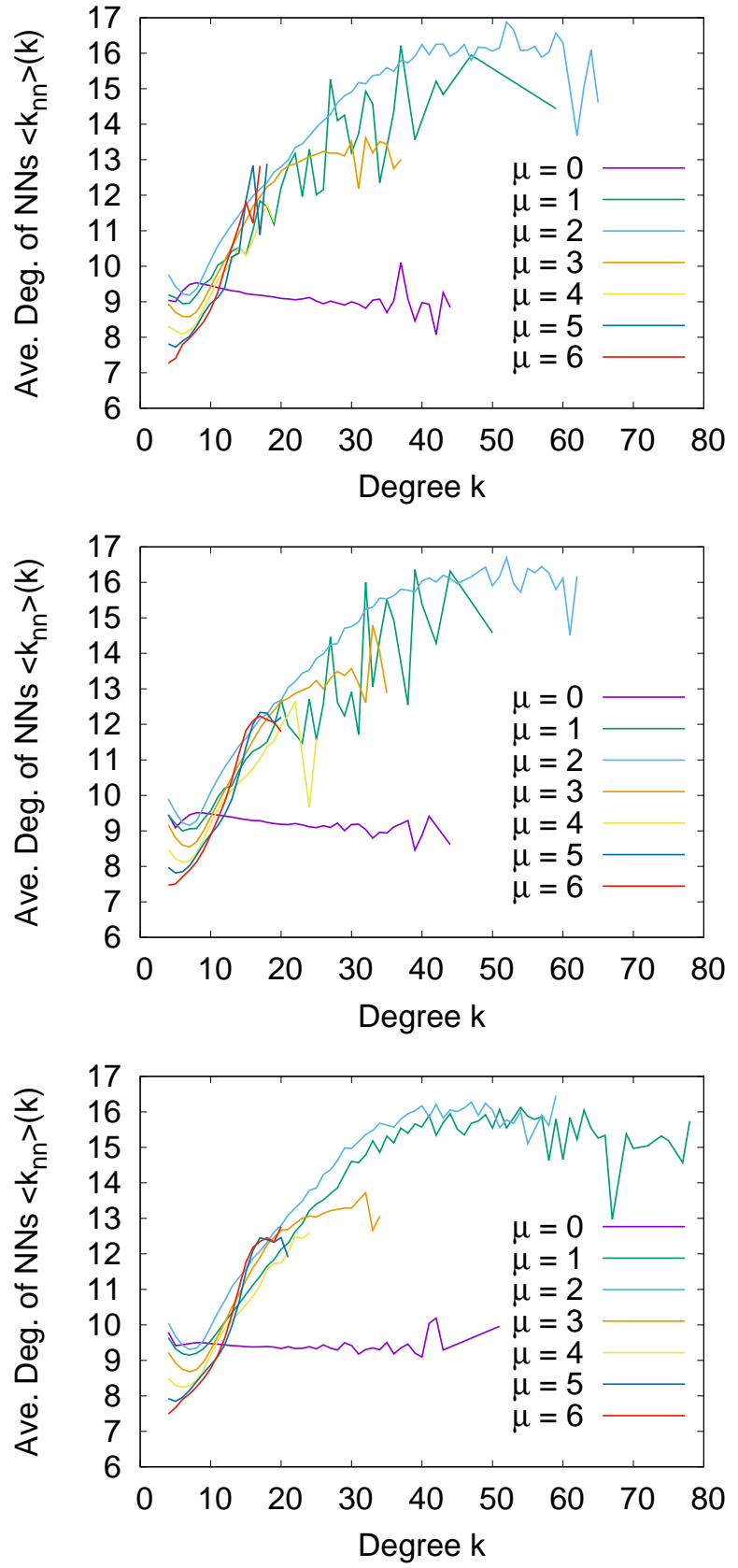

FIG. 4: ADNN in the networks at $N=7000$ with surface constraint by DLA(Top), IP(Middle), and Eden(Bottom) models.

In addition, the degree becomes medium (green) in the area out of the core, while it tend to be smaller (yellow) as closer to surface. Outer (blue) links between surface nodes appear in $\mu \geq 1$ as shown from Fig. 5(b) to (d), while it is a reason of weak robustness in the case of $\mu=0$ that outer links does not appear in Fig. 5(a). Thus, outer links contribute to enhance 
the robustness of connectivity, moderately long links are also necessary in this reason.

\section{CONCLUSION}

We have shown that onion-like topological structure with positive degree-degree correlations and strong robustness of connectivity can emerge in a spatially embedded network by pair of random and intermediation attachments even with the constraint of surface growth. In particular, it is found that moderately long links are necessary to enhance the correlations between large degree nodes at the inner core and to be onion-like. These results will be useful for designing prospective future networks for transportation or communication systems.

\section{Acknowledgment}

This research is supported in part by JSPS KAKENHI Grant Number JP.17H01729.

[1] T. Tanizawa, S. Havlin, and H.E. Stanley, "Robustness of onionlike correlated networks against targeted attacks," Physical Review E, Vol. 85, pp.046109, 2012.

[2] C.M. Schneider, A.A. Moreira, J. José S. Andrade, S. Havlin, and H.J. Herrmann, "Mitigation of malicious attacks on networks," Proc. of Nat. Acad. of Sci., Vol. 810, No. 10, pp.3838-3841, 2011.

[3] M.E. Newman, "Assortative mixing in networks," Physical Review Letters, Vol. 89, No. 20, pp.208701, 2003.

[4] Z.-X. Wu, and P. Holme, "Onion structure and network robustness," Physical Review E, Vol.81, pp.026116, 2011.

[5] Y. Hayashi, "Growing self-organized design of efficient and robust complex networks," IEEE Xplore Digital Library, Proc. of 2014 IEEE 8th Int. Conf. on SASO: Self-Adaptive and SelfOrganizing Systems, pp.50-59, 2014.

[6] Y. Hayashi, "A new design principle of robust onion-like networks self-organized in growth," Network Science, Vol. 6, No. 1, pp.54-70, 2018.

[7] T. Nishiguchi, Global Neighborhoods -Strategies of Successful Organizational Networks- (in Japanese), NTT Publishing, 2007. 
[8] R.V. Sole, R. Pastor-Satorras, E. Smith, and T.B. Kepler, "A model of large-scale proteome evolution," Advances in Complex Systems,, Vol. 5, No. 1, pp.43-54, 2002.

[9] Y. Hayashi, "Spatially self-organized resilient networks by a distributed cooperative mechanism," Physica A, Vol. 457, pp.255-269, 2016.

[10] Y. Hayashi, and N. Uchiyama, "Onion-like networks are both robust and resilient," Scientific Reports, Vol. 8, No.11241, pp.1-13, Author Correction No. 14596, 2018.

[11] A.-L. Babarási, R. Albert, and H. Jeong, "Mean-field theory for scale-free random networks," Physica A, Vol. 272, pp.173-187, 1999.

[12] T.A. Witten, and L.M. Sander, "Diffusion-limited aggregation," Physical Review B, Vol. 27, No. 9, pp.36-44, 1983.

[13] D. Wilkinson, and J.F. Willemsen, "Invasion percolation: a new form of percolation theory," Journal of Physics A: Mathematical and General, Vol. 16, pp.3365-3376, 1983.

[14] M. Eden, "A two-dimensional growth process," In: J. Neyman (Ed.), Proceedings of the 4tn Berkeley Symposium on Mathematical Statistics and Probability, Vol. IV of IV, pp.223-239, 1961. 

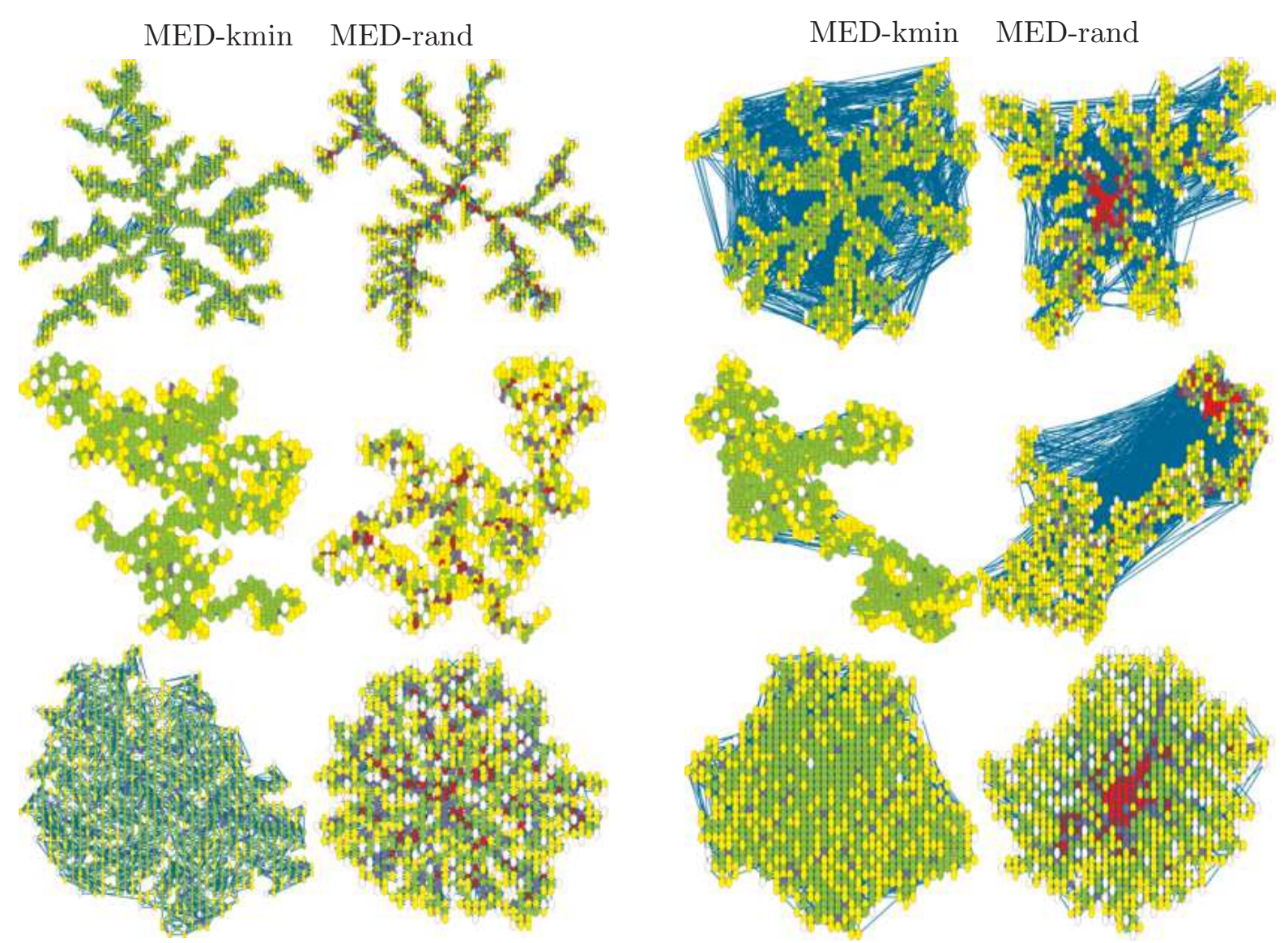

(a) $\mu=0$

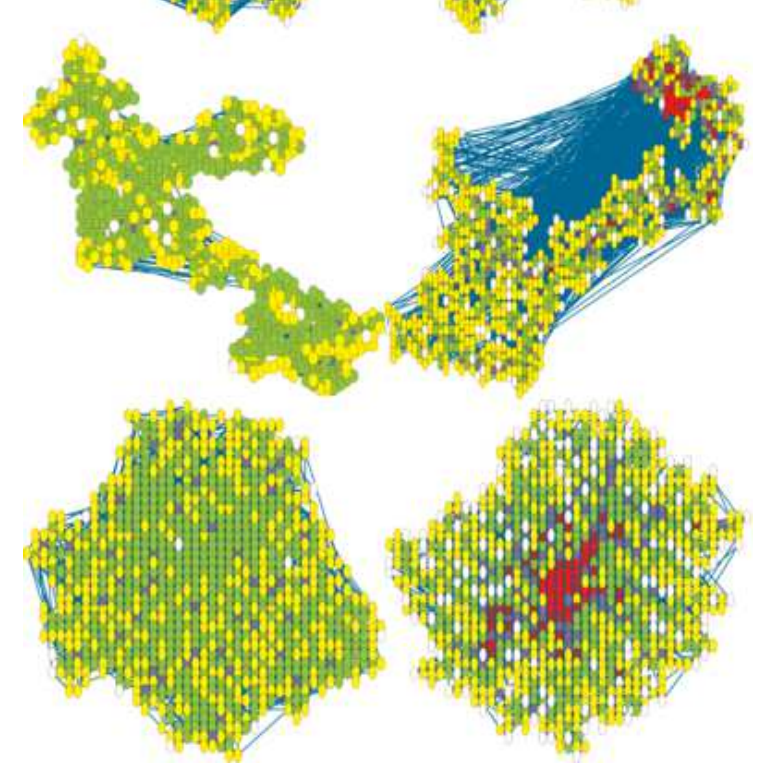

(b) $\mu=1$
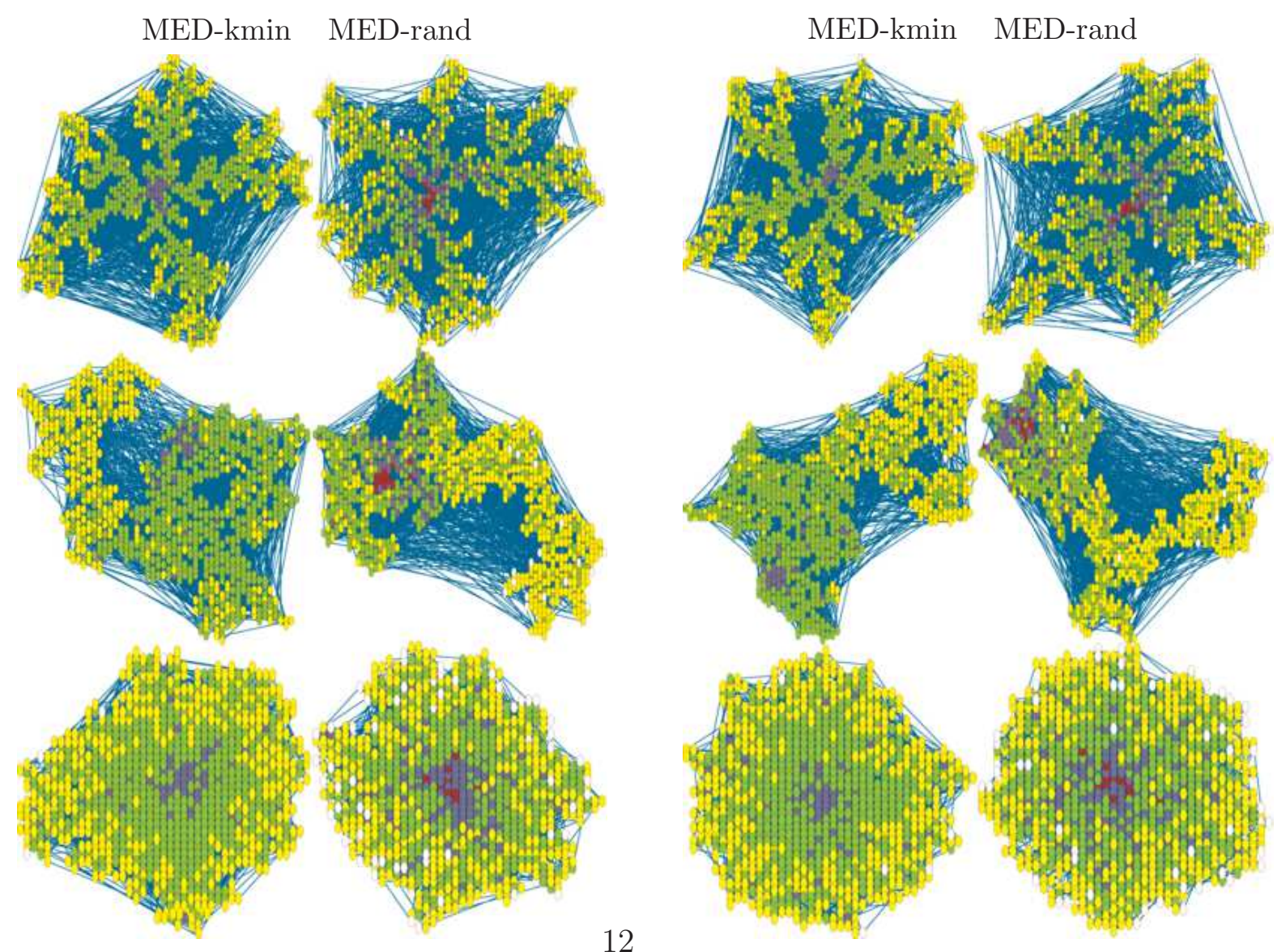

(c) $\mu=5$

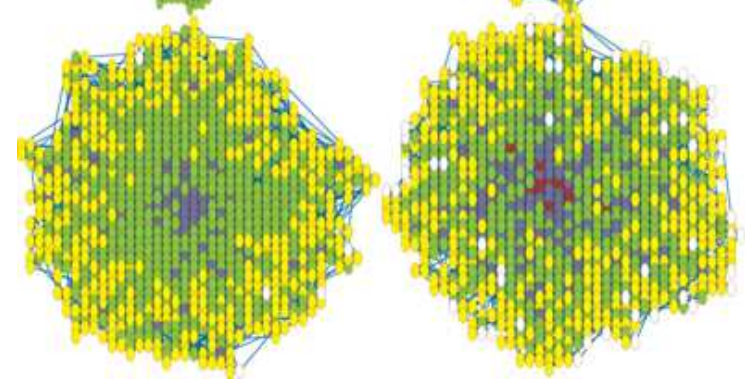

(d) $\mu=6$ 